\title{
EFEITO DA ADIÇÃO DE POLPA DE CAJU SOBRE AS QUALIDADES SENSORIAIS DE IOGURTE INTEGRAL ADOÇADO COM MEL DE ABELHA
}

\section{Effect of addition of pulp cashew on the sensory acceptance of integral yogurt sweetened with honey}

\begin{abstract}
Maiane Alves de Macedo ${ }^{I^{*}}$, Camila Carvalho Menezes ${ }^{l}$, Julianne Viana Freire Portela ${ }^{l}$, Stella Regina Sobral Arcanjo ${ }^{\prime}$, Maria Rosiane Moura ${ }^{1}$, Amanda Mazza Cruz de Oliveira ${ }^{I}$
\end{abstract}

\section{RESUMO}

A fabricação de iogurte no Brasil aumentou de maneira bastante considerável. Além de ser um produto de excelente qualidade sensorial, bastante diversificado e de boa aceitabilidade, seu consumo está relacionado com a imagem saudável e nutritiva. Neste sentido, este trabalho propôs-se elaborar um iogurte integral batido com polpa de caju e adoçado com mel de abelha que apresente boas características sensoriais, bem como determinar o valor nutricional da formulação com melhor resposta de aceitabilidade. Três amostras de iogurte com diferentes proporções de polpa de caju $(5,15$ e $25 \% \mathrm{~m} / \mathrm{m})$ e com igual percentual de mel de abelha $(15 \% \mathrm{~m} / \mathrm{m})$ foram analisadas utilizando os testes sensoriais de aceitação, ordenação-preferência e intenção de compra. Utilizou-se tabela de informação para determinar os parâmetros obrigatórios para a rotulagem nutricional da formulação mais aceita. Dentre as formulações testadas, a amostra A, com $25 \% \mathrm{~m} / \mathrm{m}$ de polpa de caju, foi a preferida com um elevado percentual de intenção de compra dos provadores, assim como as maiores médias para todos os atributos avaliados. Trata-se portanto de uma alternativa viável na agregação de valor ao caju e mel e na ampliação do mercado de laticínios.

Palavras-chave: laticínios; Anacardium occidentale L.; leite fermentado; análise sensorial.

\section{ABSTRACT}

The yogurt production in Brazil increased substantially every year. Yogurt

1 Universidade Federal do Piauí, Campus Senador Helvídio Nunes de Barros, Rua Cícero Duarte, s/n, Junco, 64600-000, Picos, PI, Brasil.E-mail: mama_macedo@hotmail.com

* Autor para correspondência 
is considered a healthy food and the diversity of this product allows great overall acceptance. This work proposed to prepare a full yogurt smoothie with cashew pulp and sweetened with honey to submit good sensory characteristics and to determine the nutritional value of the formulation with better response acceptability. Three preparations with 5,15 and $25 \% \mathrm{w} / \mathrm{w}$ of cashew pulp and $15 \% \mathrm{w} / \mathrm{w}$ of honey were evaluated with sensory analyses comprising overall acceptability, preference and purchase intent. Information table was used to determine the parameters required for nutrition labeling of the best formulation. These results showed the preparation containing $25 \% \mathrm{w} / \mathrm{w}$ of cashew pulp as the better choice by the sensory panel. The results suggest a feasible option and revels the cashew as a good material source to the dairy market.

Keywords: dairy; Anacardium occidentale L.; fermented milk; sensory analysis.

\section{INTRODUÇÃO}

O consumo de alimentos fermentados, principalmente derivados do leite como o iogurte, tem sido estimulado por profissionais de saúde, em função de seu valor nutricional e funcional. Esses alimentos fermentados apresentam proteínas de alto valor biológico e microrganismos capazes de promover diversos benefícios à saúde humana (SILVA; NASCIMENTO, 2007).

Os derivados lácteos, em geral, são submetidos a uma série de modificações tecnológicas com o objetivo de atender à diversidade da demanda do mercado consumidor. Assim, a indústria dispõe de variedade de formulações de iogurte, com diferentes sabores, consistências, aromas e cores, determinados de acordo com o processo de fabricação, matérias primas e ingredientes utilizados (THOMPSON et al., 2007).

Quanto ao sabor, o iogurte pode ser natural, adicionado de frutas, adoçado e/ ou aromatizado (THOMPSON et al., 2007). O sabor doce no iogurte pode ser obtido por meio da adição de sacarose, geleias de frutas, mel de abelha ou ainda pela adição de edulcorantes artificiais permitidos pela legislação brasileira (REIS et al., 2009). Tais ingredientes são utilizados como forma de mascarar a alta acidez do iogurte integral tradicional, que influencia negativamente a aceitação por parte de muitos consumidores, além de aumentarem o valor nutricional desses produtos lácteos (ORDÓÑEZ et al., 2005).

A Região Nordeste destaca-se como grande produtora de frutas tropicais, dentre elas o caju (Anacardium occidentale L.), produzido em grandes quantidades no estado do Ceará e Piauí. Nesse último estado, o município de Picos destaca-se como o maior pólo produtor do fruto (BONS..., 2007). A cultura brasileira do caju apresenta importância econômica na Região Nordeste, com uma produção anual de cerca de 200 mil toneladas de amêndoas e 2 milhões de toneladas de pedúnculo (PINHO, 2009). Segundo Costa et al. (2003) estima-se que a utilização do pseudofruto pela indústria é de apenas $20 \%$ ao ano, sendo direcionada principalmente para o mercado interno, com a produção de sucos e doces.

Além do caju, o Piauí destaca-se na produção do mel de abelha, com cerca de 50\% de toda produção nordestina. O município de Picos figura-se como o maior polo produtor de mel do Nordeste, sendo o segundo maior produtor do país (BONS..., 2007). O mel, além de ser um adoçante natural e fonte de energia, apresenta efeitos imunológico, antibacteriano, anti-inflamatório, analgésico, sedativo, expec- 
torante e hiposensibilizador (SOUZA et al., 2004). A apicultura é uma atividade relevante, representando uma alternativa de ocupação e renda para o homem do campo. Além disso, é uma atividade de fácil manutenção e de baixo custo inicial, em relação às demais atividades agropecuárias (FREITAS et al., 2004).

Visando encontrar alternativas tecnológicas que proporcionem a agregação de valor ao mel e ao pedúnculo do caju produzidos abundantemente na região de Picos - Piauí, este trabalho propõe elaborar um iogurte integral batido com polpa de caju e adoçado com mel de abelha que apresente boas características sensoriais, bem como determinar o valor nutricional da formulação com melhor resposta de aceitabilidade.

\section{MATERIAL E MÉTODOS}

\section{Obtenção das matérias primas}

A polpa de caju foi obtida congelada $\left( \pm-18^{\circ} \mathrm{C}\right)$, em embalagem de polipropileno com peso de $5 \mathrm{~kg}$, diretamente de produtores cooperados do Município de Santo Antônio de Lisboa - PI, as quais foram transportadas sem interrupção da cadeia de frio. Para utilização no iogurte, a polpa foi descongelada em ambiente refrigerado, a temperatura aproximada de $10^{\circ} \mathrm{C}$ durante 12 horas, sendo, posteriormente, armazenada sob temperatura de refrigeração $\left(5^{\circ} \mathrm{C}\right)$.

$\mathrm{O}$ mel de origem floral obtido em recipientes de polipropileno de 1 litro à temperatura ambiente $\left( \pm 30^{\circ} \mathrm{C}\right)$ foi doado por uma central de cooperativas apícolas da região.

O leite de vaca integral UHT (Bethânia ${ }^{\circledR}$ ) e o leite em pó integral (Embaré ${ }^{\circledR}$ ) foram obtidos no comércio local, em embalagens Tetra Pak de 1 litro e embalagem metálica flexível de $200 \mathrm{~g}$, respectivamente.

Como cultivo iniciador utilizou-se fermento em pó composto por Lactobacillus delbrueckii ssp.bulgaricus e Streptococcus salivarius ssp. thermophilus (Visbyvac DipYo-Mix VM 1-30®).

\section{Processamento do iogurte}

As formulações de iogurte integral natural foram desenvolvidas no Laboratório de Técnica e Dietética da Universidade Federal o Piauí - Campus Senador Helvídio Nunes de Barros município de Picos - PI, segundo o fluxograma proposto por Ordóñez (2005) que divide o processo em quatro fases. A primeira fase compreende recepção e controle das matérias primas, enriquecimento com sólidos lácteos, filtração, desaeração e homogeneização, tratamento térmico e resfriamento lento até $42^{\circ} \mathrm{C}$. A segunda fase é descrita como o processo de incubação (adição do cultivo iniciador). A terceira etapa é destinada a maturação do produto e a quarta compreende a fase de quebra da massa e acondicionamento.

Foram produzidas três amostras de iogurtes com proporções diferentes de polpa de caju (Tabela 1). Para cada um dos três

Tabela 1 - Concentração dos ingredientes adicionados às formulações de iogurte

\begin{tabular}{lccccc}
\hline formulação & $\begin{array}{c}\text { leite UHT } \\
(\mathrm{mL})\end{array}$ & $\begin{array}{c}\text { polpa de caju } \\
(\%)^{*}\end{array}$ & $\begin{array}{c}\text { mel de abelha } \\
(\%)^{* *}\end{array}$ & $\begin{array}{c}\text { leite em pó } \\
\text { integral }(\%)^{* * * *}\end{array}$ & $\begin{array}{c}\text { volume } \\
\text { total }(\mathrm{mL})\end{array}$ \\
\hline amostra A & 2.000 & 25 & 15 & 15 & 3306 \\
amostra B & 2.000 & 15 & 15 & 15 & 3041 \\
amostra C & 2.000 & 5 & 15 & 15 & 2777 \\
\hline
\end{tabular}

* Em relação ao iogurte integral; ** Em relação ao volume de leite e polpa; *** Em relação ao volume de leite, polpa e mel. 
tratamentos misturou-se leite UHT integral e leite em pó integral (15\% em relação ao volume de leite, polpa e mel) o qual foi utilizado como agente de corpo. Posteriormente, a mistura foi submetida a tratamento térmico a $85^{\circ} \mathrm{C}$ por 30 minutos e, em seguida, resfriada até $42^{\circ} \mathrm{C}$, para então ser adicionada a cultura láctea, na proporção de $1 \%$ em relação ao volume de leite. Procedeu-se a incubação dos tratamentos por cerca de 4 horas à temperatura de $42^{\circ} \mathrm{C}$. Após a incubação, realizou-se o resfriamento lento até $10^{\circ} \mathrm{C}$ e estocagem sob refrigeração $\left( \pm 5^{\circ} \mathrm{C}\right)$, durante 12 horas, para maturação. Depois de maturados, a massa foi quebrada e adicionada de polpa de caju $(5 \%, 15 \%$ e $25 \%)$ e mel de abelha ( $15 \%$ do volume final de leite e polpa). Em seguida, foram homogeneizados por 2 minutos utilizando batedeira elétrica, envasados e estocados sob refrigeração $\left( \pm 5^{\circ} \mathrm{C}\right)$, por um período máximo de 24 horas, até o momento das avaliações sensoriais.

\section{Análise sensorial}

A análise sensorial (testes de aceitação, ordenação-preferência e intenção de compra) foi realizada no Laboratório de Análise Sensorial da Universidade Federal do Piauí - Campus Senador Helvídio Nunes de Barros após aprovação pelo Comitê de Ética da Universidade Federal do Piauí, sob parecer $\mathrm{n}^{\mathrm{o}}$ 0457.0.045.000-10. Os testes foram conduzidos com 60 provadores selecionados, considerando-se como critérios de exclusão: indivíduos portadores de diabetes melitos e/ ou intolerantes à lactose, bem como aqueles que referiram não gostar ou não consumir caju, mel, leite e/ou seus derivados. Os voluntários assinaram o Termo de Consentimento Livre e Esclarecido respeitando as recomendações estabelecidas pela resolução 196/96 do Conselho Nacional de Saúde (BRASIL, 1996).

As amostras foram servidas sob luz branca, de forma monádica, em copos descartáveis de $100 \mathrm{~mL}$ codificados, previamente, com números aleatórios de três dígitos, contendo aproximadamente $40 \mathrm{~mL}$ de cada formulação. Foi ofertado água entre as amostras.

\section{Teste de Aceitação}

Para o teste de aceitabilidade foi utilizada escala hedônica estruturada de 9 pontos (JONES et al., 1955) como forma de avaliar os atributos aparência, aroma, cor, sabor, textura, e aceitação global. Os dados foram submetidos à análise de variância univariada (ANOVA). Em caso de diferença significativa, aplicouse o teste de Tukey para comparação entre as médias, com nível de significância de 5\%, utilizando-se o programa ASSISTAT versão 7.7 beta (OLIVEIRA et al., 2008).

\section{Teste de ordenação-preferência}

No teste de ordenação-preferência (MORAES, 1993), os julgadores foram solicitados a ordenar cada uma das três formulações desenvolvidas, conforme ordem decrescente de sua preferência. A avaliação dos resultados foi realizada pelo Teste de Friedman no qual, inicialmente, atribuiu-se a cada amostra, em cada resposta, um valor correspondente à posição em que a amostra foi ordenada (3 para a amostra mais preferida, 2 para a amostra intermediária e 1 para a amostra menos preferida).

Com o número de tratamentos testados e o número de julgamentos obtidos, calculou-se a diferença crítica entre os totais de ordenação. Tais dados foram avaliados utilizando-se a tabela de Newell e Mac Farlane (valores críticos para comparação com os módulos das diferenças entre as somas das ordens do teste de ordenação), a 5\% de significância (JONES et al., 1955).

\section{Índice de aceitação}

O índice de aceitabilidade para cada um dos atributos avaliados foi calculado de 
acordo com Teixeira et al. (1987). Para isso utilizou-se a seguinte equação:

I.A. $(\%)=($ NMA $\times 100) / N A$, Onde,

I.A. = Índice de aceitação;

NMA = Nota média do atributo;

NA $=$ Nota mais alta observada no atributo avaliado.

Os resultados foram avaliados considerando aceito o produto que apresentou índice maior ou igual a 70\% (TEIXEIRA et al., 1987).

\section{Intenção de compra}

A pesquisa da intenção de compra para cada produto elaborado foi realizada por meio de uma escala mista de cinco pontos, variando de "eu certamente compraria" a "certamente não compraria". Os resultados foram avaliados conforme a relação de frequência e considerado viável para o mercado aqueles produtos que apresentarem notas iguais ou superiores ao escore 4, ou seja "eu possivelmente compraria" (MINIM, 2010).

\section{Composição química e rotulagem nutricional}

Para a determinação da composição química (carboidrato, proteínas, gorduras totais, fibra alimentar e sódio) da amostra mais aceita e/ou preferida, utilizou-se a Tabela Brasileira de Composição de Alimentos TACO (NEPA/UNICAMP, 2006). Em seguida, foi elaborada a rotulagem nutricional, de acordo com as diretrizes vigentes da Agência Nacional de Vigilância Sanitária (ANVISA), RDC 259 (ANVISA, 2002), RDC 359 (ANVISA, 2003a) e RDC 360 (ANVISA, 2003b).

\section{RESULTADOS E DISCUSSÃO}

\section{Teste de aceitação}

Em relação às formulações de iogurtes, observou-se que os atributos aparência, aroma, textura e cor apresentaram notas acima do escore 6 "gostei ligeiramente" refletindo em boa aceitação de todas as amostras de iogurtes analisadas (Tabela 2). A aparência apresentou diferença significativa $(\mathrm{p}>0,05)$ apenas para a amostra C ( $5 \%$ de polpa de caju).

Com relação aos atributos aroma, sabor, cor e aceitação global do iogurte, a amostra A obteve médias significativamente maiores que as amostras B e C, as quais não apresentaram diferença estatisticamente significativa entre si. O maior percentual de polpa de caju $(25 \%)$ conferido a amostra A pode ter sido o fator determinante de tal diferença, devido a acentuação do aroma, sabor e cor característicos do fruto. Silva et al. (2008) avaliaram sensorialmente

Tabela 2 - Escores médios de aceitação para os atributos aparência, aroma, sabor, textura, cor e aceitação global (AG) das formulações de iogurtes com diferentes concentrações de polpa de caju e adoçados com mel

ATRIBUTOS SENSORIAIS

AMOSTRAS

\begin{tabular}{lcccccc}
\hline & Aparência & Aroma & Sabor & Textura & Cor & AG \\
\hline A (25\% de polpa) & $7,6^{\mathrm{a}}$ & $7,15^{\mathrm{a}}$ & $7,23^{\mathrm{a}}$ & $7,65^{\mathrm{a}}$ & $7,6^{\mathrm{a}}$ & $7,08^{\mathrm{a}}$ \\
B (15\% de polpa) & $7,26^{\mathrm{a}}$ & $6,31^{\mathrm{b}}$ & $5,25^{\mathrm{b}}$ & $7,43^{\mathrm{a}}$ & $7,45^{\mathrm{b}}$ & $5,68^{\mathrm{b}}$ \\
C (5\% de polpa) & $7,03^{\mathrm{b}}$ & $6,08^{\mathrm{b}}$ & $5,13^{\mathrm{b}}$ & $7,33^{\mathrm{a}}$ & $7,2^{\mathrm{b}}$ & $5,33^{\mathrm{b}}$ \\
DMS & 0,57 & 0,80 & 0,90 & 0,63 & 0,56 & 0,86 \\
\hline
\end{tabular}

* Médias seguidas pela mesma letra na coluna não diferem entre si pelo teste de Tukey a 5\% de significância.

* Diferença mínima significativa. 
formulações de néctar de caju adoçado com mel de abelha e observaram que o teor de suco nas formulações foi determinante na preferência dos consumidores, sendo que as médias aumentaram progressivamente em direção às formulações com maior teor de suco.

Em estudo realizado por Oliveira (2003), observou-se efeito significativamente positivo da adição de sacarose e do aumento do teor de sólidos totais do leite, bem como da cultura empregada na firmeza do iogurte. Desta forma, a adição de mel, assim como o aumento do teor de sólidos totais pelo uso do leite em pó pode ter contribuído de forma expressiva para respostas positivas em relação à textura para todas as formulações analisadas. Essas não apresentaram diferença estatisticamente significativa entre si. E, caracterizaram-se por textura homogênea, suave e consistente.

Estudo de bebida fermentada (SANTOS et al., 2012) também verificaram que o aumento na concentração do fruto jenipapo, interferiu positivamente para a aceitação global do produto, resultando em respostas acima do escore 6.

\section{Teste de ordenação-preferência}

Os resultados do teste ordenaçãopreferência das formulações de iogurte desenvolvidas (Tabela 3 e Figura 1) corroboram as respostas de aceitação discutidas anteriormente. Quanto maior a quantidade de polpa de caju adicionada à formulação (amostra A), maiores foram as respostas de aceitação e de preferência entre os provadores. A amostra $A$ foi estatisticamente diferente com relação às amostras B e C.

Tabela 3 - Totais das somas de ordenaçãopreferência para as amostras de iogurtes com diferentes concentrações de polpa de caju e adoçados com mel

\begin{tabular}{cc}
\hline AMOSTRAS & Soma das Notas* \\
\hline A $(25 \%$ de polpa $)$ & $162^{\mathrm{a}}$ \\
B (15\% de polpa $)$ & $104^{\mathrm{b}}$ \\
C (5\% de polpa $)$ & $94^{\mathrm{b}}$ \\
\hline
\end{tabular}

* Notas seguidas pela mesma letra não diferem entre si a 5\% de significância, considerou-se como diferença crítica, valores iguais ou maiores que 26 entre os totais, segundo Teste de Fridman (NEWELL; MAC FARLANE, 1987)

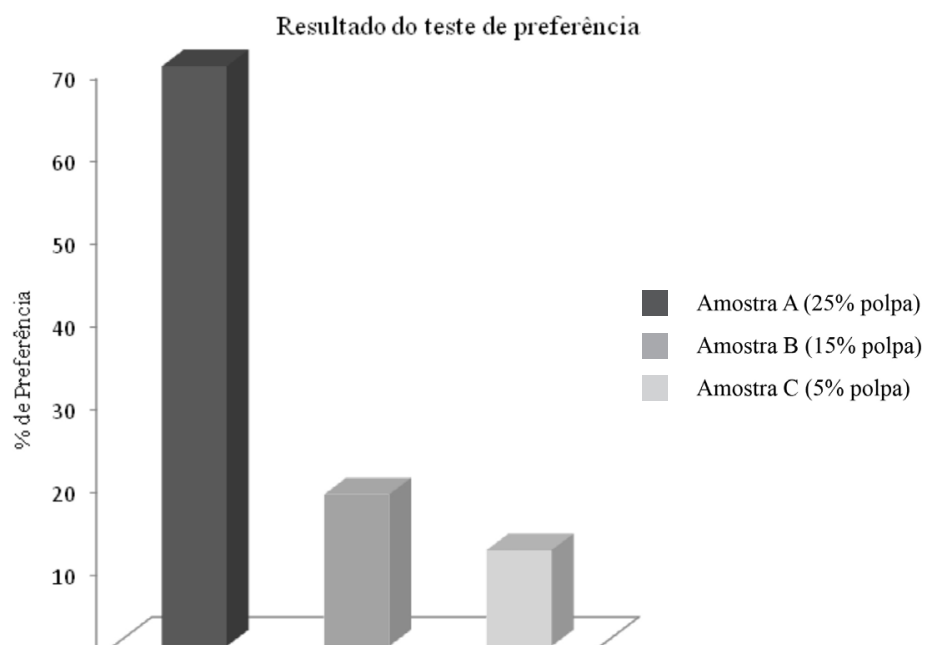

Figura1 - Resultado do teste de preferência para as três amostras de iogurtes com diferentes concentrações de polpa de caju e adoçados com mel 


\section{Índice de aceitação}

Quanto maior a quantidade de polpa de caju adicionada à formulação, maiores foram os índices de aceitação para todos os atributos avaliados. Isso indica que tais respostas sensoriais foram intensificadas pela concentração do fruto utilizado no processamento, uma vez que este pseudofruto é bastante apreciado na região de estudo. Somente os atributos sabor e aceitação global para as amostras B e C, e o aroma para a amostra $\mathrm{C}$ não apresentaram resultados satisfatórios, uma vez que, Teixeira et al. (1987) indica que índices acima de 70\% refletem positivamente na aceitação do produto por parte do consumidor (Tabela 4).

Em estudo realizado por Rocha et al. (2008), no qual foram adicionados frutos do cerrado ao iogurte, observou-se também boa aceitação por parte dos consumidores, pela utilização de frutos típicos da região que in natura já apresentavam grande aceitabilidade entre a população. Altos índices também foram alcançados para sobremesas lácteas sabor manga, cajá e mangaba (CLEMENTINO et al., 2006) e para leite fermentado adicionado de jenipapo (SANTOS et al., 2012) corroborando a boa aceitação por produtos lácteos oriundos de frutos tropicais.

\section{Intenção de compra}

Os resultados da intenção de compra em relação aos produtos avaliados revelaram que a atitude de compra dos consumidores para a amostra $\mathrm{A}$ é superior às demais amostras (Figura 2). Isso corrobora com as respostas sensoriais de aceitação e preferência mencionadas nos itens anteriores, nas quais as maiores influências foram dos atributos aroma, sabor e cor. Verifica-se, portanto, que ao condensar os conceitos "certamente compraria" e "provavelmente compraria", ou seja, igual ou acima do escore 4 , a amostra A ( $25 \%$ de polpa de caju) obteve-se percentuais acima de $75 \%$ de intenção de compra positiva. Isso confirma o obtido em amostras de leite fermentado sabor jenipapo (SANTOS et al., 2012)

\section{Composição química e rotulagem nutricional}

Considerando as respostas sensoriais, $\mathrm{e}$ constatando-se que a amostra A ( $25 \%$ polpa $)$ foi a formulação mais aceita e preferida dentre as analisadas, procedeu-se a análise da sua composição nutricional e desenvolvimento da rotulagem nutricional (Tabela 5). Essa foi elaborada de acordo com as diretrizes da Agência Nacional de Vigilância Sanitária

Tabela 4 - Índice de aceitação por atributos avaliados das amostras de iogurte com diferentes concentrações de polpa de caju e adoçado com mel de abelha

\begin{tabular}{cccc}
\hline & \multicolumn{3}{c}{ INDICE DE ACEITAÇÃO (\%) } \\
\hline ATRIBUTO & $\begin{array}{c}\text { Amostra A } \\
(25 \% \text { polpa })\end{array}$ & $\begin{array}{c}\text { Amostra B } \\
(15 \% \text { polpa })\end{array}$ & $\begin{array}{c}\text { Amostra C } \\
(5 \% \text { polpa })\end{array}$ \\
\hline Aparência & 84,4 & 80,6 & 78,1 \\
Aroma & 79,4 & 70,1 & 67,5 \\
Sabor & 80,3 & 58,3 & 57,0 \\
Textura & 85,0 & 82,5 & 81,4 \\
Cor & 85,0 & 82,8 & 80,0 \\
Aceitação Global & 78,7 & 63,1 & 59,2 \\
\hline
\end{tabular}


(ANVISA), RDC 259 (ANVISA, 2002), RDC 359 (ANVISA, 2003a) e RDC 360 (ANVISA, 2003b), com o auxílio da Tabela Brasileira de Composição de Alimentos (NEPA/UNICAMP, 2006).

A formulação apresentou valor energético consideravelmente alto (282 kcal por porção) o que representa $14 \%$ do valor diário de referência, provavelmente, devido à adição de leite em pó integral e mel de abelha à formulação. A RDC 359 (ANVISA, 2003) que dispõe sobre porções de alimentos embalados para fins de rotulagem nutricional de alimentos, preconiza um valor energético médio de 125 kcal para porção de leite e derivados $(200 \mathrm{~mL})$. Em estudo realizado por Rodas et al.

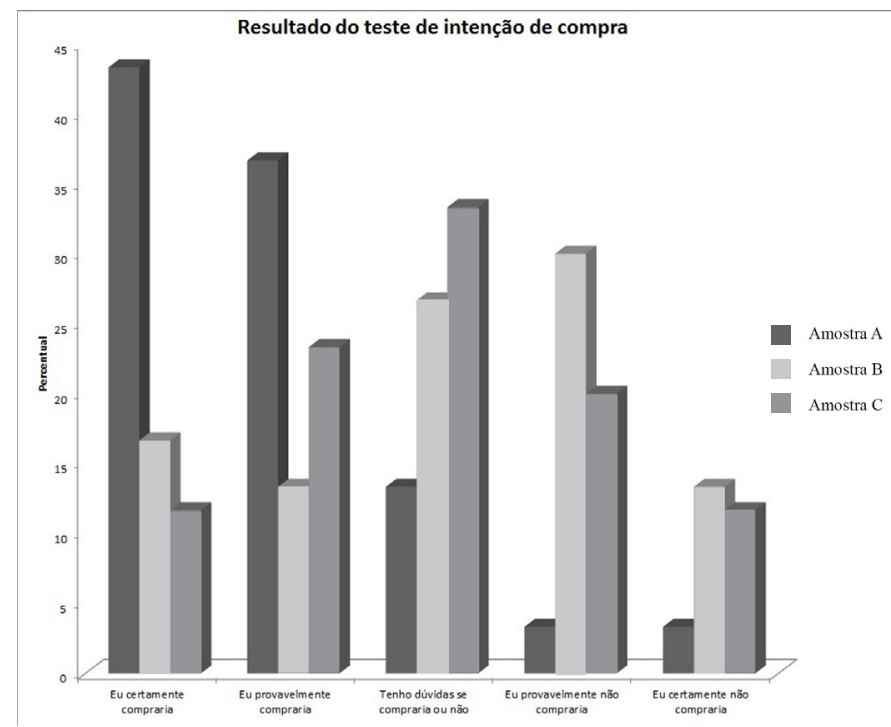

Figura 2 - Resultado do teste de intenção de compra para as três amostras de iogurtes com diferentes concentrações de polpa de caju e adoçados com mel

Tabela 5 - Informação nutricional para $200 \mathrm{~mL}$ da formulação de iogurte integral batido com $25 \%$ de polpa de caju e adoçada com mel de abelha

\begin{tabular}{lcc}
\hline & $\begin{array}{c}\text { Quantidade por porção } \\
\text { Porção de 200mL (1 copo) }\end{array}$ & VD* $(\%)$ \\
\hline Valor Energético & $282 \mathrm{kcal}=1384 \mathrm{~kJ}$ & 14 \\
Carboidrato $(\mathrm{g})$ & 38 & 13 \\
Proteína (g) & 10 & 14 \\
Gorduras totais $(\mathrm{g})$ & 10 & 19 \\
Gorduras saturadas $(\mathrm{g})$ & $* *$ & $* *$ \\
Gorduras trans $(\mathrm{g})$ & $* *$ & VD não estabelecido \\
Fibra Alimentar $(\mathrm{g})$ & 0,2 & 1 \\
Sódio (mg) & 164 & 7 \\
\hline
\end{tabular}

* Valores Diários de referência com base em uma dieta de 2000 kcal ou 8400 kJ

**Valores não calculados 
(2001) no qual foram caracterizados iogurtes com frutas de diferentes marcas comerciais, observou-se que o valor calórico dos mesmos variou significativamente entre $76 \mathrm{kcal} / 100 \mathrm{~g}$ e $118 \mathrm{kcal} / 100 \mathrm{~g}$. Esses valores estão abaixo do referenciado no presente estudo.

Ainda segundo Rodas et al. (2001) os valores percentuais de carboidratos e lipídios sofrem alterações significativas entre marcas, enquanto que o valor proteico dos iogurtes não diferem significativamente entre si. As variações na composição química do iogurte podem ser atribuídas tanto ao teor lipídico do leite utilizado (integral, semidesnatado ou desnatado), como à variedade de ingredientes que podem ser adicionados na fabricação do iogurte como a adição de sólidos e edulcorantes, o que justifica os valores encontrados no presente estudo. Os teores de proteína, carboidratos e lipídios determinados no presente estudo são semelhantes aos de algumas das marcas comercias de iogurte analisadas por Rodas et al. (2001).

Como os cálculos da composição nutricional da amostra foram baseados na concentração de ingredientes e em valores descritos na Tabela Brasileira de Composição de Alimentos - TACO (NEPA/UNICAMP, 2006), os dados apresentados podem não retratar fielmente a composição da amostra, pois pode superestimar ou subestimar um ou outro nutriente. Portanto, sugere-se uma análise mais específica da composição nutricional do produto.

\section{CONCLUSÕES}

Além de constituir-se em um produto rico em nutrientes que pode promover benefícios à saúde, o iogurte com maior percentual de polpa de caju apresentou adequadas características sensoriais e grande percentual de intenção de compra pelos consumidores. Esse produto pode ser uma alternativa para agregar valor ao iogurte, mel de abelha e pedúnculo de caju, esses dois últimos produtos típicos da Região Nordeste. Portanto, constitui-se em uma alternativa para a indústria alimentícia no Brasil.

\section{REFERÊNCIAS}

AGÊNCIA NACIONAL DE VIGILÂNCIA SANITÁRIA (ANVISA). Resolução RDC nº 259, de 20 de setembro de 2002 alterada pela Resolução RDC $\mathrm{n}^{0} 123$, de 13 de maio de 2004 e complementada pela resolução RDC n ${ }^{0} 163$, de 17 de agosto de 2006. Aprova o Regulamento Técnico sobre Rotulagem de Alimentos Embalados. Diário Oficial da República Federativa do Brasil, Brasília, 23 set. 2002.

AGÊNCIA NACIONAL DE VIGILÂNCIA SANITÁRIA (ANVISA). Resolução RDC n ${ }^{\circ} 359$, de 23 de dezembro de 2003. Aprova Regulamento Técnico de Porções de Alimentos Embaladospara Fins de Rotulagem Nutricional.Diário Oficial da República Federativa do Brasil, Brasília, 26 dez. 2003a.

AGÊNCIA NACIONAL DE VIGILÂNCIA SANITÁRIA (ANVISA). Resolução RDC n³60, de 23 de dezembro de 2003 complementada pela Resolução RDC n 163, de 17 de agosto de 2006. Aprova Regulamento Técnico sobre Rotulagem Nutricional de Alimentos Embalados, tornando obrigatória a rotulagem nutricional.Diário Oficial da República Federativa do Brasil, Brasília, 26 dez. 2003b.

BRASIL. Ministério da Saúde. Conselho Nacional de Saúde. Resolução n.196, de 10 de outubro de 1996. Diretrizes e normas regulamentadoras de pesquisas envolvendo seres humanos. Diário Oficial da República Federativa do Brasil, Brasília, 10 out. 1996.

CLEMENTINO, I. M.; NASCIMENTO, J. do, CORREIA, R. T. P. Sobremesa láctea aerada tipo mousse produzida a partir de leite de caprino e frutas regionais. Revista PublICa, v. 3, n.1, p. 1-8, 2007.

COSTA, T. S. A.et al. Determinação de tanino em pedúnculo de caju: método da valinina versus método do butanol ácido. Química Nova, v.26, n.5, p. 763-765, 2003.

FREITAS, D. G. F.et al. Nível tecnológico e 
rentabilidade de produção de mel de abelha (Apis Mellifera) no Ceará. Revista de Economia e Sociologia Rural, v. 42, n.1, p.171-188, 2004.

JONES, L.V.; PERYAM, D.R.; THURSTONE L.L. Development of a scale for measuring soldiers food preferences. Food Research, v. 20, n.5, p. 512-520, 1955.

MINIM, V. de P. R. Análise Sensorial: estudos com consumidores. 2 ed. Viçosa: Editora UFV, 2010. 308p.

MORAES, M. A. C. Métodos para avaliação sensorial dos alimentos. 8. ed. Campinas: Editora da UNICAMP, 1993.78p.

NÚCLEO DE ESTUDOS E PESQUISAS EM ALIMENTAÇÃO- UNICAMP (NEPA/UNICAMP). Tabela brasileira de composição de alimentos TACO. Versão 2. Campinas: NEPA/UNICAMP, 2006. 113 p.

NEWELL, G. J.; MACFARLANE, J. D. Expanded tables for multiple comparison procedure on the analysis of ranked data. Journal of Food Science, v.52, n.6, p.1721-1725, 1987.

OLIVEIRA, K. A. M.etal.Desenvolvimento de formulação de iogurte de araticum e estudo da aceitação sensorial.Alimentos e Nutrição, v.19, n.3, p. 277-281, 2008.

OLIVEIRA, M. N.et al. Efeito do teor de sólidos e de sacarose na acidificação, firmeza e viabilidade de bactérias do iogurte e probióticas em leite fermentado. Ciência e Tecnologia de Alimentos, v.23 (supl.), p. 172-176, 2003.

ORDÓÑEZ, J. A. et al. Tecnologia de Alimentos: alimentos de origem animal.Porto Alegre: Artmed, 2005.v. 2, 280p.

PINHO, L. X. Aproveitamento do resíduo do pedúnculo de caju (Anacardium occidentale L.) para a alimentação humana. 2009. 99f. Dissertação (Mestrado em Ciência e Tecnologia de Alimentos) - Universidade Federal do Ceará, Fortaleza, 2009.
REIS, J. S.et al. Impacto da utilização de diferentes edulcorantes na aceitabilidade de iogurte "light" sabor morango. Alimentos e Nutrição, v. 20, n.1, p. 53-60, 2009.

BONS negócios no semiárido. Revista Horizonte Geográfico, n. 113, 2007. Disponível em: < http:// horizontegeografico.com.br/exibirMateria/175> Acesso em: 10 out. 2013.

ROCHA, C.et al. Elaboração e avaliação de iogurte sabor frutos do cerrado. Boletim do Centro de Pesquisa de Processamento de Alimentos, v.26, n.2, p.255-266, 2008.

RODAS, M. A. B.et al. Caracterização físicoquímica, histológica e viabilidade de bactérias lácticas em iogurtes com frutas. Ciência e Tecnologia de Alimentos, v. 21, n.3, p.304-309, 2001.

SANTOS, G.et al. Avaliação sensorial, físicoquímica e microbiológica do leite fermentado probiótico desnatado adicionado de Jenipapo desidratado osmoticamente. Revista do Instituto de Laticínios Cândido Tostes, v. 67, n. 388, p. 61-67, 2012.

SILVA, A. R.et al. Néctar de caju adoçado com mel de abelha: desenvolvimento e estabilidade. Ciência e Tecnologia de Alimentos, v.28, n.2, p.348-354, 2008.

SILVA, E. B.; NASCIMENTO K. O. Avaliação da adequação da rotulagem de iogurtes. Ceres: Nutrição \& Saúde, v.2, n.1, p. 9-14, 2007.

SOUZA, R. C. S.et al. Valor nutricional do mel e pólen de abelhas sem ferrão da região amazônica. Revista Acta Amazônica, v. 34, n. 2, p. 333-336, 2004.

TEIXEIRA, E.et al. Análise Sensorial de Alimentos. Florianópolis: UFSC, 1987.182 p.

THOMPSON, J. L. et al. Preferences for commercial strawberry drinkable yogurts among African American, Caucasian and Hispanic consumers in the United States. Journal of Dairy Science, v.90, n.11, p. 4974-4987, 2007. 